\title{
Harnessing catalysis to enhance scanning probe nanolithography
}

Cite this: Nanoscale, 2014, 6, 4998

\begin{abstract}
Stewart A. M. Carnally and Lu Shin Wong*
The use of scanning probes bearing catalysts to perform surface nanolithography combines the exquisite spatial precision of scanning probe microscopy with the synthetic capabilities of (bio)chemical catalysis. The ability to use these probes to direct a variety of localised chemical reactions enables the generation of nanoscale features with a high degree of chemical complexity in a "direct-write" manner. This article surveys the range of reactions that have been employed and the key factors necessary for the successful use of such catalytic scanning probes. These factors include the experimental parameters such as write speed, force applied to the probes and temperature; as well as the processes involved in the preparation of the catalysts on the probes and the surface that is to be fabricated. Where possible, the various reactions are also compared and contrasted; and future perspectives are discussed.
\end{abstract}

Received 2nd February 2014 Accepted 23rd March 2014

DOI: $10.1039 / c 4 n r 00618 f$

www.rsc.org/nanoscale removal of ablation of material ("destructive" nanolithography). A wide variety of materials have been directly written or templated using these techniques including polymers, colloidal nanoparticles (NPs), carbon nanotubes, sol-gel precursors, small organic molecules, biomolecules (proteins and oligonucleotides) and even single virus particles and bacteria. ${ }^{6,7}$

However, all the methods above essentially rely on the physical manipulation of materials and surfaces by the probe tip. As an alternative, there has also been interest in combining the high spatial control of scanning probe microscopy with the synthetic capabilities of (bio)chemical catalysts, to directly perform chemical reactions at the point of contact between the probe and the surface in order to generate nanoscale features. A straightforward example of this concept is the use of DPN to deliver catalytically reactive inks where the deposited catalyst mediates a reaction with the surface to produce a feature. ${ }^{11-14} \mathrm{~A}$ more direct approach to catalytic scanning probe nanolithography (cSPL) is to employ a scanning probe that is itself rendered catalytically active. In principle, direct cSPL would offer advantages by avoiding the need to deposit the catalyst as an ink, thus avoiding issues related to the transport and diffusion of the ink on the surface, and allowing the catalyst to be repeatedly reused (although not indefinitely, see Section 6 below). Examples of catalytic probes include the application of a transition metal coating, ${ }^{15-20}$ or more rarely, functionalisation of the probes with metal complexes, ${ }^{21}$ organic catalysts, ${ }^{22}$ photocatalysts $^{23,24}$ or enzymes. ${ }^{25-29}$

This article will focus on the types of catalytic probes that have been reported and discuss the physicochemical aspects of lithography, as well as its influence on catalysis. Where possible, comparisons are drawn between cSPL-based strategies and those that involve other related methods such as DPN and microcontact printing $(\mu \mathrm{CP})$. 


\section{Transition metal-catalysed nanolithography}

\subsection{Hydrogenations}

The first example of catalytic scanning probe lithography was reported in 1995 and employed a Pt-coated AFM probe as a hydrogenation catalyst. ${ }^{15}$ Here, the probe was scanned over an azidosilane self-assembled monolayer (SAM) under $\mathrm{H}_{2}$ saturated isopropanol, resulting in the reduction of the terminal azides to amines (Fig. 1A). The surface feature generated was then treated with amine-specific reagents and fluorescent labels to confirm the azide reduction (Fig. 2A). A tip loading force of $400 \mathrm{nN}$ was required to achieve catalysis (lower loading forces are described only as producing 'decreased' levels of catalytic reduction) at a "writing" speed of $1 \mu \mathrm{m} \mathrm{s}^{-1}$. The loading force was chosen as it represented the force required to penetrate probe/sample solvation layers.

Transfer hydrogenations using formic acid as the hydrogen donor have been subsequently reported using Pd-coated probes. ${ }^{16}$ When this type of hydrogenation was applied to azide reduction, pattern formation occurred at tip-loading forces of 3.0-4.6 $\mu \mathrm{N}$ at speeds up to $5 \mu \mathrm{m} \mathrm{s}^{-1}$, although trace levels of

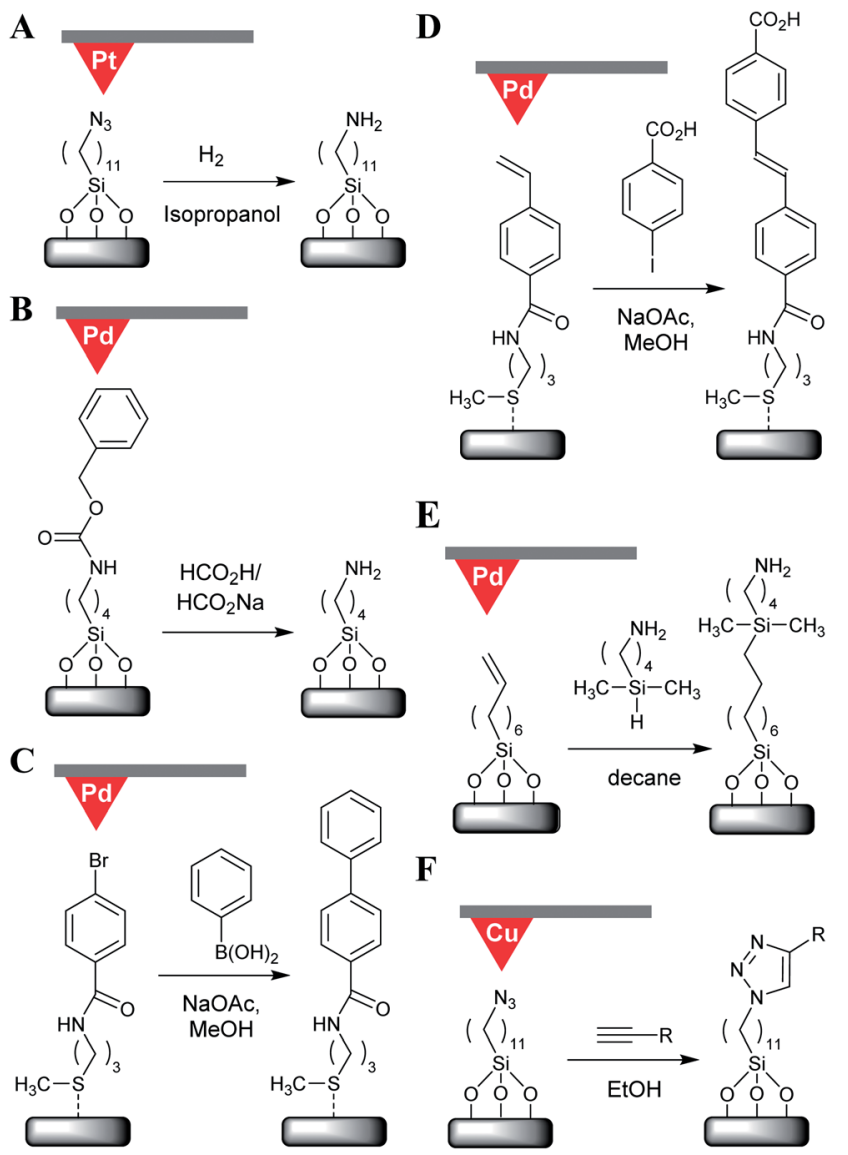

Fig. 1 Representative chemical reaction schemes for catalytic scanning probe nanolithography employing metallic probes: (A) azide reductions; (B) CBz removal by debenzylation; (C) Suzuki and (D) Heck cross-couplings; (E) hydrosilylation; (F) CuAAC 'click' ligations.
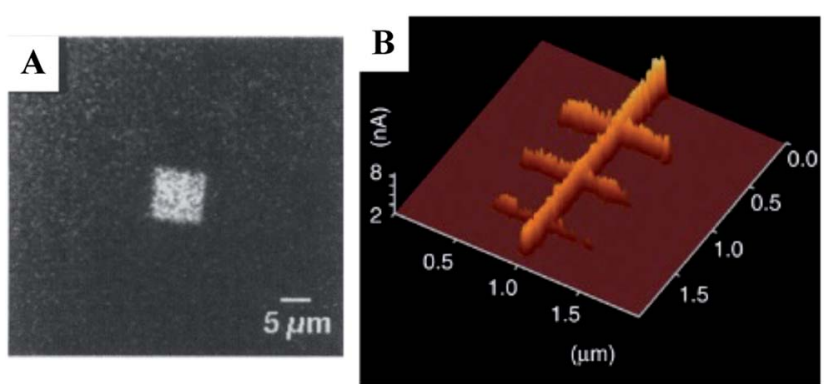

Fig. 2 Example images generated by scanning probe-catalysed hydrogenations: (A) fluorescence microscopy image of a $10 \times 10 \mu \mathrm{m}^{2}$ feature generated on an azide-functionalised surface after scanning probe mediated reduction and labelling with an amine-reactive fluorophore; (B) conductive AFM image of reduced GO features on a bulk GO surface, demonstrating increased electrical conductivity in the reduced areas. The smallest feature width is $20 \mathrm{~nm}$ (line on lower left of image). Reprinted with permission from AAAS and Nature Publishing Group.

catalysis were still detectable by fluorescent labelling of terminal amines at up to $10 \mu \mathrm{m} \mathrm{s}^{-1}$. When applied for the removal of a benzyloxycarbonyl (Cbz) protecting group from an aminosilane SAM (Fig. 1B), this reaction required loading forces above $2.5 \mu \mathrm{N}$ at $1.5 \mu \mathrm{m} \mathrm{s} \mathrm{s}^{-1}$, with no upper speed threshold specified. Under these conditions, both transfer hydrogenation reactions resulted in features with line widths in the $30-40 \mathrm{~nm}$ range.

These experiments suggest that larger forces are needed for transfer hydrogenation compared to the use of molecular hydrogen, which may be related to the sensitivity of transfer hydrogenations towards altered temperatures and pressures, ${ }^{30}$ both of which will be elevated at the point of contact between the probe and surface (see Section 6 below). However, any such effect is also convoluted by the fact that larger forces may be acting to compensate for the faster write speeds employed in these cases.

Probe catalysed thermally induced hydrogenations have also gathered interest more recently in their application to the nanolithography of reduced graphene oxide (rGO) nanoribbons from graphene oxide (GO) using a Pt-coated AFM probe. ${ }^{20}$ Here, the sample was heated to $115{ }^{\circ} \mathrm{C}$ under a $\mathrm{H}_{2}$ atmosphere $(0.1$ $\mathrm{MPa}$ ), with lithography successfully performed at tip loading forces of 1-10 $\mathrm{nN}$ and speeds of $2-10 \mathrm{~nm} \mathrm{~s}^{-1}$; with the depth and diameters of the written features being proportional to the loading force, and inversely proportional to write speed. Under optimum conditions features as narrow as $20 \mathrm{~nm}$ were reported (Fig. 2B). Notably, this nanolithography method resulted in features that were $\sim 0.5 \mathrm{~nm}$ deep despite the fact that the presented hydroxyl groups and epoxide bridges would only be expected to account for a height difference of $0.1-0.2 \mathrm{~nm}$. This discrepancy is likely to result from height added to the GO surface though a water layer bound to oxygen-containing groups that are absent in the rGO nanoribbons.

Temperature also proved a critical factor in this method of lithography, with pattern formation beginning at $50{ }^{\circ} \mathrm{C}$, improving substantially at $100{ }^{\circ} \mathrm{C}$ to reach an optimum at 115 
${ }^{\circ} \mathrm{C}$ (thought to be due to the decreasing prevalence of adsorbed water with elevated temperature). Above $115{ }^{\circ} \mathrm{C}$ contrast in electrical conductivity between the rGO features and the surrounding GO declines due to partial thermo-chemical reduction of GO. As an alternative approach, thermochemical nanolithography (TCNL) of GO using an unmodified, heated probe (as opposed to the cSPL approach wherein the entire sample was heated) has been demonstrated. ${ }^{31}$

The thermochemical approach required loading forces an order of magnitude greater (120-230 $\mathrm{nN}$ vs. 1-10 $\mathrm{nN})$ and considerably higher temperatures $\left(\sim 600-1200{ }^{\circ} \mathrm{C}\right)$ than the cSPL method. The cSPL approach also achieves conductivities in the rGO nanoribbons 30-fold greater than TCNL, with 2-3 orders of magnitude greater conductivity contrast between the reduced and oxidised regions (cSPL: $\mathrm{rGO}=10^{5} \mathrm{~S} \mathrm{~m}^{-1} v s$. GO $=$ $10^{-5}$ to $10^{-3} \mathrm{~S} \mathrm{~m}^{-1}$; TCNL: $\mathrm{rGO}=10^{3} \mathrm{~S} \mathrm{~m}^{-1}$ vs. $\mathrm{GO}=10^{-1} \mathrm{~S}$ $\mathrm{m}^{-1}$ ). Although the thermochemical lithography was performed under $\mathrm{N}_{2}$ rather than $\mathrm{H}_{2}$, bulk studies on the bulk uncatalysed reduction of GO under $\mathrm{Ar} / \mathrm{H}_{2}$ show that temperatures of 800$1000{ }^{\circ} \mathrm{C}$ are required for effective reduction. ${ }^{32}$

Taken together, these data clearly show that the cSPL approach to GO reduction occupies an entirely different parametric space from TCNL (Fig. 3).

\subsection{Carbon-carbon and carbon-silicon bond-forming reactions}

Transition metal catalysis has been extended to carbon-carbon bond formation and several examples cSPL using Pd-catalysed couplings have been described. ${ }^{17,18,33}$ In the first example of this concept, biaryl groups were written on an aryl bromide-presenting gold-alkylthiolate SAM by Suzuki coupling (Fig. 1C). ${ }^{17}$ The probes used in this report were coated with polyvinyl-pyrrolidone (PVP)-coated palladium nanoparticles (PdNP), and required forces of $20-25 \mathrm{nN}$ (at $1 \mu \mathrm{m} \mathrm{s}^{-1}$ ) to initiate catalysis. By increasing the scanning speed (to $40 \mu \mathrm{m} \mathrm{s}^{-1}$ ) whilst lowering the tip loading force (1-5 $\mathrm{nN}$ ) the same probe can be used to image the sample non-reactively. A subsequent report ${ }^{18}$ then demonstrated writing of 10-15 $\mathrm{nm}$ biphenyl-amine features on to arylbromide SAMs, requiring $15-20 \mathrm{nN}$ at $1 \mu \mathrm{m} \mathrm{s} \mathrm{s}^{-1}$ for catalysis, although some patterning could still be achieved at writing speeds up to $8 \mu \mathrm{m} \mathrm{s}^{-1}$. This report then extended to demonstrate a probe-directed Heck reaction coupling $p$-iodobenzoic acid to a styrene-presenting SAM (Fig. 1D), achieving a resolution down to $13 \pm 3 \mathrm{~nm}$. Here, catalysis required forces of $25-40 \mathrm{nN}$ at $2 \mu \mathrm{m} \mathrm{s}^{-1}$, with patterning declining sharply above $3 \mu \mathrm{m} \mathrm{s}^{-1}$.

Subsequent detailed analysis ${ }^{33}$ revealed that the nature of the Pd coating strongly influenced the performance and lifetime of the probe. Sputter-coated Pd proved incapable of any detectable lithography, electrodeposited Pd irreversibly lost activity after patterning approximately $10^{6}$ turnovers while PVP-PdNP probes were the most robust; although these also typically irreversibly lost activity after $10^{9}$ turnovers (patterning of 10 's $\mu \mathrm{m}^{2}$ ). The limited lifespan was postulated to be due to Ostwald ripening of the Pd surfaces whereby small PdNPs coalesce into larger particles, or rougher surfaces smoothen, resulting in loss of the reactive high-energy surfaces.

It was also found that inverting the Heck coupling (the surface presenting the aryl halide SAM and the styrene in the solution phase) was found to reduce the reaction turnover 3-4 fold. This observation was proposed to be due to the transfer of the rate-determining step (oxidative addition of aryl halide to Pd) from solution phase to the surface, resulting in a retardation of this step due to bulk steric hindrance from the surface. In the Suzuki coupling, changing the SAM's terminal halide from bromine to iodine lowered turnover (from $\sim 2.1 \times 10^{4}$ to $1.4 \times 10^{4} \mathrm{~s}^{-1}$ ), which may result from aryl bromides having a more negative activation volume than aryl iodides (despite $\mathrm{C}-\mathrm{Br}$ bonds being stronger than $\mathrm{C}-\mathrm{I}$ ), hence are more responsive to the elevated pressure beneath the probe.

PdNP-catalysed Heck reactions have also been demonstrated using catalytic $\mu \mathrm{CP},{ }^{34}$ where the PdNPs were prepared from phase-separated block-copolymer lithography. Features as small as $15 \mathrm{~nm}$ could be produced using this catalyticallyenabled nanolithography method, which provides a notable alternative strategy to cSPL.

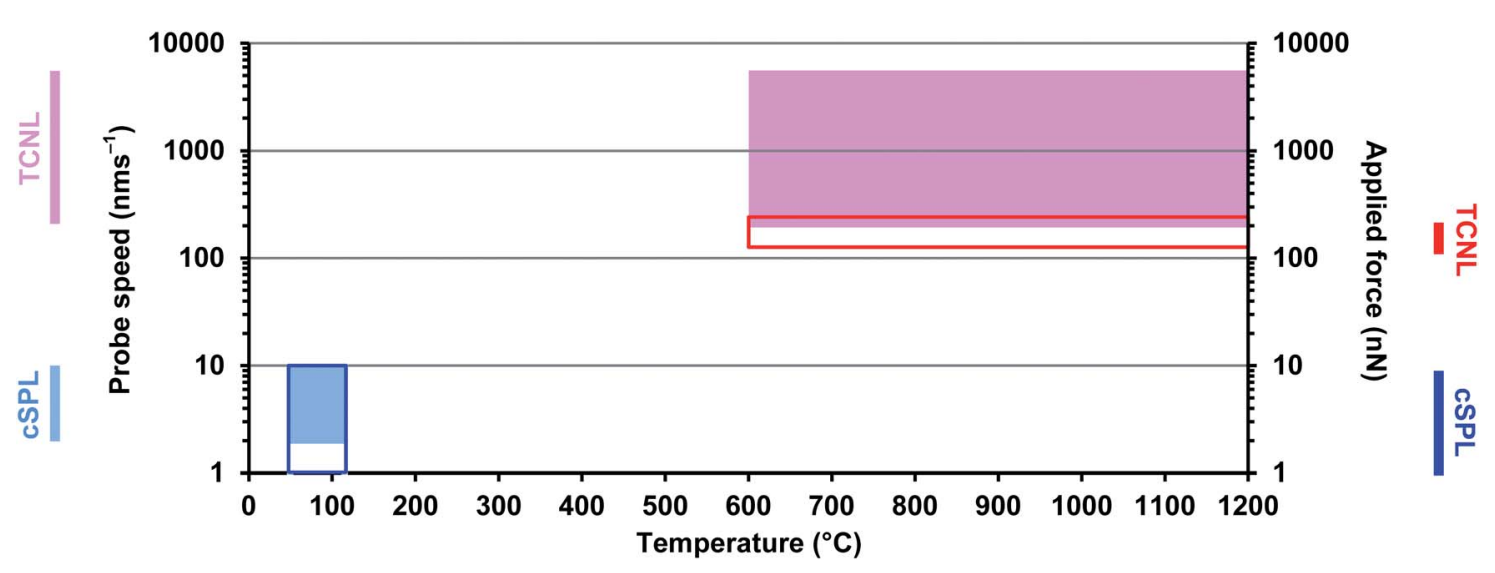

Fig. 3 Graph of lithography speed and applied probe force against temperature showing the regions where GO reduction occurs. The filled light blue area indicates the probe speed for CSPL, the pink area indicates those speeds associated with successful TCNL. The dark blue outline box indicates the force applied for CSPL and the red box for TCNL. 
In terms of carbon-silicon bond formation, hydrosilylations involving the addition of aminobutyldimethylsilane to the terminal alkene of octenylsiloxane films (Fig. 1E) have been found to require $2.5 \mu \mathrm{N}$ of force and could be performed at speeds up to $5 \mu \mathrm{m} \mathrm{s}^{-1}$ to give line widths of $\sim 60 \mathrm{~nm} .{ }^{16}$ Pd-catalysed hydrosilylation of terminal alkenes has also been demonstrated using PdNPs immobilised to a $\mu$ CP stamp ${ }^{35}$ with a H-terminated Si surface and the alkene as the ink. In this case, a pattern resolution of $20 \mathrm{~nm}$ was reported but required $20 \mathrm{~min}$ of contact; 6-7 orders of magnitude longer than the ms contact times associated with cSPL hydrosilylation.

\subsection{Copper-catalysed 'click' ligations}

The copper(I)-catalysed azide-alkyne cycloaddition (CuAAC) 'click' reaction has been employed to catalytically lithograph surfaces using Cu catalyst immobilised on to AFM probes ${ }^{19}$ and $\mu \mathrm{CP}$ stamps, ${ }^{36}$ or deposited as ink in with scanning probes ${ }^{13,37-39}$ and $\mu \mathrm{CP} .^{36}$

In the cSPL approach, ${ }^{19}$ a $\mathrm{Cu}$-coated AFM probe was employed to click a range of alkynes to an azidosilane SAM (Fig. 1F), achieving a minimal line width of $50 \mathrm{~nm}$ (close to the expected diameter of the metal coated probe). Tip loading forces of 260-300 $\mathrm{nN}$ (at $2 \mu \mathrm{m} \mathrm{s}^{-1}$ ) proved optimal for catalysis, with higher forces displacing the SAM. It is notable that in comparison to solution phase click reactions that require a reducing agent to produce $\mathrm{Cu}(\mathrm{I})$ in situ from a $\mathrm{Cu}$ (II) salt, it appears that sufficient $\mathrm{Cu}(\mathrm{I})$ is present in the native oxide layer of the probe's $\mathrm{Cu}$ coating for catalysis without the need for in situ reduction.

In contrast, there are many examples of DPN-based strategies where the probe deposits a soluble copper catalyst. The first reported example used an AFM probe inked with $\mathrm{CuI}$ and an azido-functionalised dendron to write features on to an alkyneterminated silane surface, which produced written line widths of $300 \mathrm{~nm} .{ }^{13}$ In other examples, the catalytically active $\mathrm{Cu}(\mathrm{I})$ ions were produced in situ by using an ink formulation containing $\mathrm{CuSO}_{4}$ and the reducing agent ascorbic acid. ${ }^{37,38}$ Together with a small molecule azide (e.g. a fluorophore or biotin), features with a width of approximately $85 \mathrm{~nm}$ could be produced (Fig. 4A). The use of in situ $\mathrm{Cu}$ (II) generation has also been applied to PPL-deposited inks, which offer the advantage of large-area lithography but with the drawback of poorer feature resolution (191 nm reported). ${ }^{39}$ Thus, feature resolution is poorer with freely diffusing catalytic ink compared to when the catalyst is immobilized to a probe, where catalysis occurs only directly beneath the probe.

\subsection{Alkene epoxidation}

There is currently only one reported example of this type of reaction, which also represents the only current example of "homogenous" catalysis in cSPL. ${ }^{21}$ This method employs Mn coordinated to 1,3,7-triazacyclononane (tacn) ligands bound to the probe for epoxidation of alkene-terminated SAMs under a solution of $\mathrm{H}_{2} \mathrm{O}_{2}$ (Fig. 5A). Writing was performed between 200$600 \mathrm{nN}$ force loads and speeds up to $4.8 \mu \mathrm{m} \mathrm{s} \mathrm{s}^{-1}$. Writing produced lines 150-200 nm wide, many times the probe diameter. The reason for this relatively poor resolution remains
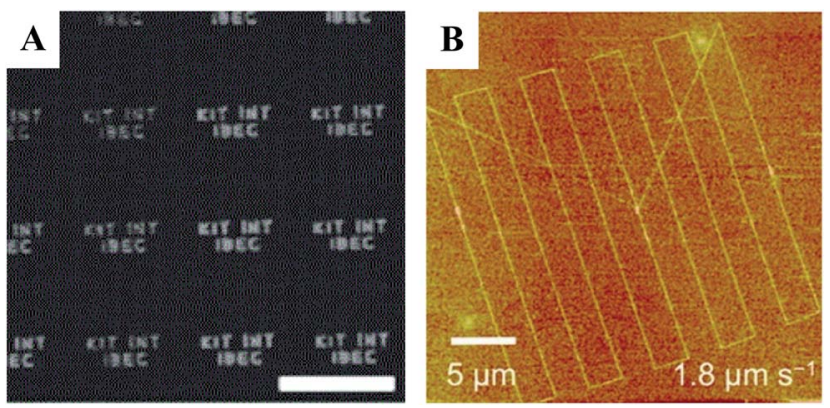

Fig. 4 (A) A fluorescence microscopy image of a fluorophore-azide ink deposited by dip-pen nanolithography. The use of an array of probes enabled the parallelised writing of multiple features simultaneously. The scale bar represents $35 \mu \mathrm{m}$. (B) Lateral force microscopy images of features formed on OEG-silane after probe-directed photooxidation followed by conjugation of aminobutylnitrilotriacetic acid. The smallest line width is $75 \mathrm{~nm}$. Reprinted with permission from John Wiley \& Sons and ACS Publications.
A
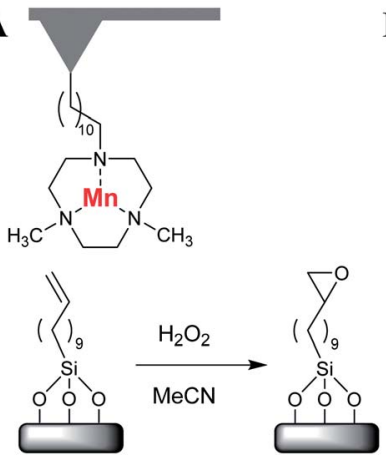

B
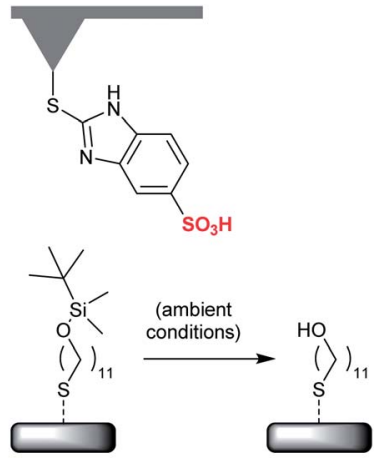

Fig. 5 Representative chemical reaction schemes for catalytic scanning probe nanolithography employing tethered catalysts: (A) Mncatalysed olefin epoxidation and; (B) sulfonic acid-catalysed deprotection.

unclear and may be due to the local release of free manganese into the water meniscus that forms between the probe and substrate under ambient conditions ( $c f$. DPN). It was also noteworthy that writing at $1.2-2.5 \mu \mathrm{N}$ resulted in both loss of the substrate SAM and irreversible loss of the probe's catalytic activity, presumably through mechanical erosion of the probeimmobilised Mn(tacn) groups.

\section{Photocatalysis}

There have been two of examples of scanning probe-directed photocatalysis. This method relies on the use of $\mathrm{TiO}_{2}$-coated probes, which under UV excitation catalyses the oxidation of adsorbed atmospheric water to generate hydroxide radicals. The underlying chemical reaction for this mode of cSPL is thus not via direct interaction with a catalyst but with an intermediate product. In the first example of this approach, ${ }^{23} \mathrm{TiO}_{2}$-coated AFM probes were used to pattern features on a surface loaded with microcrystals of the azo dye Procion Red MX-5B. Repeated scanning with $\mathrm{TiO}_{2}$ probe under a UV light source $(254 \mathrm{~nm})$ 
resulted in loss of surface roughness and changes in the UV-vis and IR spectra of the surface, which were used to infer successful catalysis. The process was slow, reaching a plateau of $\sim 70 \%$ decrease in roughness after $6 \mathrm{~h}$ of repeated scanning over the same $30 \times 30 \mu \mathrm{m}^{2}$ area.

In a further refinement to the basic concept, $\mathrm{TiO}_{2}$ coated AFM probes and SNOM (scanning near-field optical microscopy) probes coated on their front face were used to photocatalytically pattern gold-alkylthiolate and silane SAMs. ${ }^{24}$ In the case of the SNOM probes, UV light (325 nm) was directed down the aperture of the probes, thus photocatalytically oxidising the thiol group in a dodecanethiol SAM, allowing replacement with 11-mercaptoundecanoic acid in what could be considered an example of light-driven nanografting. In this report, lateral force microscopy imaging demonstrated a $\sim 90 \mathrm{~nm}$ resolution.

The same researchers also demonstrated the use of "apertureless" photocatalytic lithography using conventional probes coated with $\mathrm{TiO}_{2}$. Here, a beam of light from an HeCd laser is directed on to the apex of the probes. These probes were used to pattern an oligoethylene-presenting silane SAM by oxidative degradation of ethylene glycol units, resulting in the formation of terminal aldehydes (Fig. 4B). ${ }^{24}$ Subsequent immobilisation of a fluorescent protein (via aminobutylnitrilotriacetic acid and nickel affinity tagging) allowed the generation of $\sim 70 \mathrm{~nm}$ fluorescent patterns. In this case, continuous lines were written at $1.8-2.5 \mu \mathrm{m} \mathrm{s}^{-1}$, with discontinuous lines observed at $4.0 \mu \mathrm{m} \mathrm{s}^{-1}$.

\section{Non-metal chemocatalysis}

In comparison with metal-mediated catalysis, there are fewer examples of organocatalysis in cSPL. The main examples in this category use a tethered strong organic acid to perform probelocalised acid-catalysed reactions. For example, Au-coated probes functionalised with 2-mercapto-5-benzimidazole sulfonic acid have been shown to remove tert-butyldimethylsilyl (TBS) protecting groups from a gold-alkylthiolate SAM to reveal the hydroxy group (Fig. 5B). ${ }^{22}$ Pattern formation was performed at tip loading forces below $10 \mathrm{nN}$ (with no minimum force specified), with catalysis confirmed by AFM topographic measurements of the reacted SAM. In this case, it is likely that the condensation of a water meniscus at the point of contact between the probe and the surface may facilitate the protonolysis. Nevertheless, the smallest line produced was about 25 $\mathrm{nm}$, which implied that the resolution of the structures was limited only by the contact area beneath the AFM tip (and possible drift in the piezoelectric scanner).

The catalytic deprotection of TBS-terminated gold-alkylthiolate SAMs by an immobilized sulfonic acid (2-mercaptoenthane sulfonic acid) has also been performed by $\mu \mathrm{CP}$, where deprotection was extended to tert-butyloxycarbonyl (Boc)-protected thiols. ${ }^{\mathbf{4 0 , 4 1}}$

\section{Enzymatic nanolithography}

\subsection{Biocatalytic inks}

In parallel with transition metal and small molecule catalysis, there has also been significant interest in the use of enzymes with scanning probe nanolithography. Such biocatalysts are attractive since they offer highly efficient catalysis in terms of yields, regio- and stereospecificity; together with their ability to promote reactions under mild conditions.

Thus far, the majority of biocatalytic nanolithographies use the probe to deposit free enzyme on to a substrate-presenting surface via DPN or related methods. Subsequent enzymatic digestion of the underlying substrate results in the formation of the nanopatterns (Fig. 6A). This concept has been demonstrated using a number of enzyme systems, including the DPN deposition of DNase I on to a single-stranded DNA monolayer, into which trenches equal in depth to the thickness of the monolayer could be generated with a lateral resolution down to $\sim 100 \mathrm{~nm} .^{11}$ In other examples, the deposition of proteinase $\mathrm{K}$ on to poly-Llactic acid films ${ }^{\mathbf{1 4}}$ resulted in features of 400-500 $\mathrm{nm}$ (after a 24 $\mathrm{h}$ incubation at $37^{\circ} \mathrm{C}$, Fig. 6B), while PPL deposition of Lipase B on $\operatorname{poly}(\varepsilon$-caprolactone) films to generated micrometre-scale features (after $7 \mathrm{~d}$ incubation at $37^{\circ} \mathrm{C}$ ). ${ }^{42}$ In all these cases, the lateral resolution was many fold larger than the probe width, again providing an illustration of the limitations of freely diffusible catalytic inks.

There have also been reports of using nano-fountain pen probes to deliver trypsin solutions. This proteolytic enzyme, when written on to a bovine serum albumin-coated surface enabled the generation of channels $\sim 100 \mathrm{~nm}$ in width under optimum conditions. ${ }^{\mathbf{4 3 , 4 4}}$

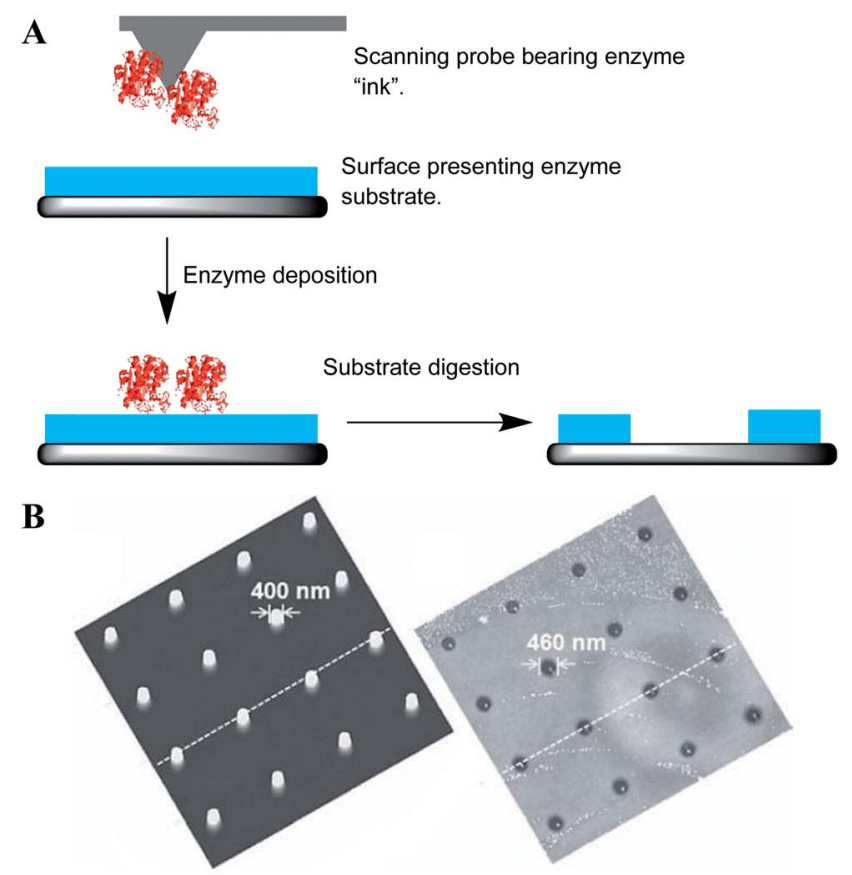

Fig. 6 (A) Schematic diagram of enzymatic nanolithography through the deposition of enzymes by scanning probes. (B) AFM topography images proteinase $\mathrm{K}$ nanopatterns on poly-L-lactic acid films deposited by DPN (left) and the corresponding biodegraded holes after washing of the films to remove the enzyme (right). Based on the data from the respective dotted lines, the features were $117 \mathrm{~nm}$ high or 11.6 $\mathrm{nm}$ deep. Reprinted with permission from John Wiley \& Sons. 
However, these examples all essentially describe destructive nanolithography, and necessitates that the surface must first be processed so that it presents the enzyme substrate. As an alternative, enzymatic DPN has also been used in constructive lithography through the deposition of materials on an arbitrary surface to generate features. In this regard, a technique has been reported for the generation of metallic nanowires via DPN deposition of metal NP-conjugated enzymes. ${ }^{12}$ Here, glucose oxidase (GOx) or galactose oxidase (GalOx) was multiply decorated with $1.4 \mathrm{~nm}$ AuNPs and deposited in lines by DPN. The patterned enzyme-NP conjugates were subsequently exposed to their substrates (glucose or galactose) and $\mathrm{HAuCl}_{4}$. The $\mathrm{H}_{2} \mathrm{O}_{2}$ generated by the enzyme then acts as a reducing agent to drive $\mathrm{Au}$ deposition on to the attached NPs, which increase in size until the enzyme becomes coated by the metal and loses activity. The growing AuNPs also merge into nanowires; $800 \mathrm{~nm}$ wide and $220 \mathrm{~nm}$ high for GOx, $500 \mathrm{~nm}$ wide and $300 \mathrm{~nm}$ high for GalOx. A similar process was performed with alkaline phosphatase (AP)-AuNP conjugates, forming a silver nanowire 500 $\mathrm{nm}$ wide and $35 \mathrm{~nm}$ high, via reduction of $\mathrm{AgNO}_{3}$ by a hydroquinone by-product of $p$-aminophenylphosphate produced by AP. This approach provides a route to the nanolithography of electrically conducting nanowires, but at the expense of lateral resolution since the growth of the NPs results in line widths typically 10 -fold larger than the originally deposited enzymeAuNP lines.

\subsection{Biocatalytic probes}

The immobilisation of the enzyme on to a probe or stamp negates the requirement for the ink and thus the need to replace a consumable or increase the complexity of the apparatus by the addition of an ink delivery system (microchannels or reservoirs). Furthermore, it ensures that catalysis takes place only at the point of probe-substrate contact. Biocatalytic lithographies with immobilised enzymes typically rely on covalent attachment of the enzyme to the stamp or probe, ${ }^{25,28,45}$ but may also use non-covalent approaches, namely the biotinstreptavidin interaction ${ }^{27,29}$ or through metal affinity with genetically encoded polyhistidine "His-tags". ${ }^{46}$

Biocatalytic nanolithography with tip-immobilised enzymes for destructive nanolithography was first demonstrated in $2003 .^{25}$ In this report, endoproteinase V8 was covalently immobilised by bioconjugation to a probe functionalised with succinimide active esters. The enzyme is known to cleave polypeptide chains at $\mathrm{C}$-termini of glutamic acid residues, and could thus be used in combination with a gold-alkylthiolate SAM composed of the peptide A(AEAAKA) ${ }_{6}$ C. Slow scanning of the enzyme-bearing probe across the monolayer $\left(50 \mathrm{~nm} \mathrm{~s}^{-1}\right.$, roughly $1 / 20^{\text {th }}$ imaging speed) with the contact force at $<50 \mathrm{pN}$ resulted in successful lithography. No lithography was observed at the usual imaging speeds of $1 \mu \mathrm{m} \mathrm{s}^{-1}$.

This basic approach was then extended to peptide-coated surfaces where the peptides were labelled with a fluorophore and quencher on either side of the cleavage site (Fig. 7A). Quenching of the fluorophore was relieved upon cleavage at the glutamic acid residue and release of the quencher, resulting in fluorescence from the hydrolysed regions. ${ }^{26,28}$ Notably, the generation of fluorescent features (i.e. successful lithography) only occurred when the probe was first etched with a focused ion beam to remove a large part of the apex of the cantilever, leaving a truncated pyramid presenting a triangular surface on to which the enzyme was attached. Using a fixed loading force of $1.2 \mathrm{nN}$, no fluorescence was observed with a probe that had not been etched. The need for this $\sim 10000$ fold increase in probesubstrate contact area provided by the etching $\left(\sim 600 \mathrm{~nm}^{2}\right.$ for the unmodified probe, $\sim 11 \mu \mathrm{m}^{2}$ for the etched probe) strongly suggested that the immobilised enzymes were poorly active. Indeed, a subsequent investigation of enzyme activity by comparing the similar amounts of the enzyme immobilised on to silicon nanoparticles using the same bioconjugate chemistry and the free enzyme revealed that V8 retained only $\sim 20 \%$ activity following covalent immobilisation.

It is interesting to note that the same research team attempted to immobilise His-tagged V8, at either the $\mathrm{N}$ - or Ctermini of the protein, on to nitrilotriacetic acid-modified AFM probes, but found that activity was lost in both cases. Addition of a His-tag to the N-terminus would be expected to abolish activity, as the $\mathrm{N}$-terminal valine interacts directly with active site of the enzyme. The C-terminus, however, is on the opposite side of the molecule, and a His-tag here would be expected to present the enzyme to the substrate at a near-ideal orientation. The reasons for this observation remain unclear but may have implications for future attempts at improving immobilised enzyme activity though control of orientation.

Probe immobilised enzymes have also been used to demonstrate constructive nanolithography. This concept was first demonstrated with AP immobilised on to probes. ${ }^{27}$ When the enzyme is treated with a mixture of 5-bromo-4-chloro- $3^{\prime}$ -

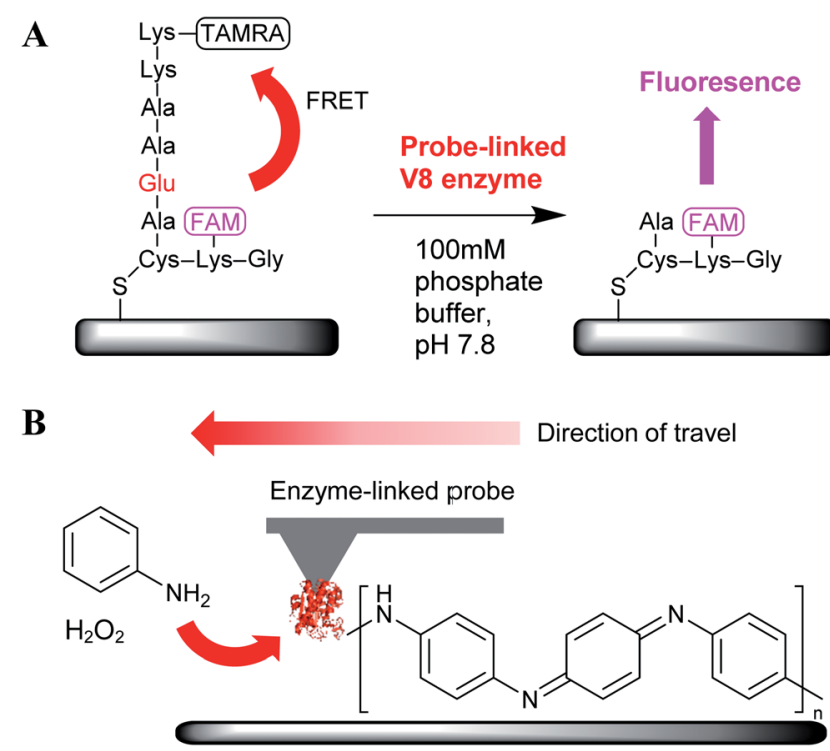

Fig. 7 Examples of scanning probe nanolithography employing enzyme-bearing probes for (A) peptide hydrolysis by proteinase enzymes; (B) oxidative polymerisation by peroxidase enzyme. FRET = fluorescence resonance energy transfer; FAM = carboxyfluorescein (fluorophore); TAMRA = tetramethylrhodamine (quencher). 

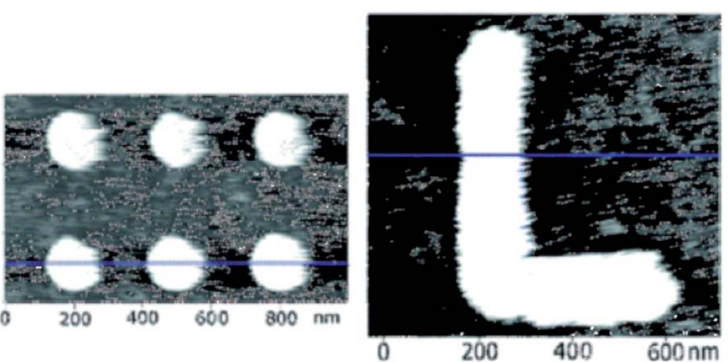

Fig. 8 Illustrative AFM topography images showing features consisting of precipitated NBT following nanolithography with a probebound AP enzyme. Based on the data from the respective blue lines, the features were approximately $150 \mathrm{~nm}$ in diameter. Reprinted with permission from ACS Publications.

indolyphosphate (BCIP) and nitro-blue tetrazolium (NBT), dephosphorylation of BCIP reduces NBT to insoluble NBT diformasan. The nanolithography is thus performed by the precipitation of the reaction product as the probe is traced across the surface, with lateral resolutions of $150-170 \mathrm{~nm}$ being reported (Fig. 8).

A further development in this area was the use of probeimmobilised horseradish peroxidase (HRP) for the nanolithographic deposition of polyaniline, a flexible, biocompatible and electrically conductive polymer. ${ }^{29}$ Here, the HRP catalysed the oxidative polymerisation of aniline with $\mathrm{H}_{2} \mathrm{O}_{2}$ (Fig. 7B). This report is particularly notable as although the deposition of NBT diformasan was an interesting proof of concept, the material used to form the features was of little use for further application. Instead, the generation of nanoscale polyaniline patterns of arbitrary geometry may prove a step towards the fabrication of biosensors, implants and nanoelectronics.

In both cases, attachment of the enzyme only to the apex of the probe was critical for such constructive lithographies since the presence of the enzyme on any other part of the probe would result in the deposition of the material across the entire surface of the probe. In order to confine the attachment of the enzyme only to the apex of the probe, the entire probe was first functionalised with biotin, a small molecule biological cofactor that is strongly bound by the protein streptavidin. The probe is then scanned across a surface bearing the enzyme-streptavidin conjugate, resulting in the 'pick-up' of the enzymes only at the point of contact.

\section{Rationalisation of lithography parameters}

Under optimum conditions cSPL is capable of patterning resolutions comparable those achieved by the other scanning probe lithographies $(<50 \mathrm{~nm})$. In contrast to methods involving the deposition of inks containing catalysts, tip loading force is the parameter most critical to successful cSPL, with catalysis routinely described as occurring only within a specific 'force window'. In general, cSPL is usually performed at higher loading forces and lower probe velocities than those used for imaging, but the interplay between speed, force applied and temperature is complex.

The moving catalyst must make and maintain physical contact with the substrate, penetrating any solvation layers present, and maintain a sufficient contact area to catalyse features of detectable width (often the presence of features must be inferred through subsequent elaboration, typically with a bulky molecular species or fluorophore). In examples using metallic or small molecule catalysts, the upper force threshold does not represent a level of force inhibitory to catalysis, but is the force above which mechanically displaces the substrate from the surface ( $>70 \mathrm{nN}$ for alkylthiolate SAMs on gold, $>400$ $\mathrm{nN}$ for siloxanes). ${ }^{16-18,21}$ At forces approaching this threshold catalysis and displacement will occur concurrently, thus the ideal force for pattern formation must lie significantly below that required for displacement.

At the nanoscale contact areas involved, the application of tip loading forces in the $\mathrm{nN}-\mu \mathrm{N}$ range have been calculated to generate GPa local pressures. ${ }^{16,33}$ Furthermore, the tip-substrate frictional (shear) forces, and the temperature at the site of catalysis may be significantly higher than room temperature and contribute to the acceleration of local chemical reactions. Experiments using $\mu \mathrm{CP}$ stamps with immobilised PdNPs required a temperature of $130{ }^{\circ} \mathrm{C}$ and $30 \mathrm{~min}$ contact time induce Heck coupling reactions ${ }^{34}$ whilst AFM-immobilised PdNPs performed the reaction without external heating and with only millisecond catalyst-substrate contact times. ${ }^{18}$ However, any rate enhancement provided by friction alone is insufficient to compensate for the reduction in contact duration accompanying increased writing speed, as feature size is universally observed to be inversely proportional to writing speed. This observation suggests that at low speeds, increased catalyst-substrate contact time will offset reductions in pressure/temperature; hence there are no reports of minimal writing speeds.

Enzymatic CSPL requires much slower speeds than CSPL with non-enzyme catalysts with $<0.3 \mu \mathrm{m} \mathrm{s}^{-1}$ and $1-5 \mu \mathrm{m} \mathrm{s}^{-1}$ respectively for the cases discussed above, although due to wide range of reactions presented it is difficult to make direct headto-head comparisons. Enzyme turnover rates are typically orders of magnitude higher than non-enzyme catalysts, however the size of enzymes will necessarily mean that the probe apex will present considerably fewer catalytically active species than a metal-coated probe. For example, a probe with a radius of curvature of $10 \mathrm{~nm}$ may be considered to end in a half spherical cap with a surface area of $\sim 628 \mathrm{~nm}^{2}$. If this were a Pt-coated probe (assuming a van der Waals radius of $0.175 \mathrm{~nm}$ for Pt and ideal surface packing), it would present $\sim 6000 \mathrm{Pt}$ atoms on its surface. If this was the same probe decorated with V8 proteinase, $\sim 32$ enzymes would occupy the same area. Assuming the probe fully penetrates a $1.5 \mathrm{~nm}$ thick substrate monolayer (an approximation for both an azidosilane and a 10mer peptide), that equates to $\sim 1000 \mathrm{Pt}$ atoms or 5 proteinase molecules in contact with the substrate. The cSPL of azidosilanes using a Pt probe ${ }^{15}$ was performed at $1 \mu \mathrm{m} \mathrm{s}^{-1}$ and peptide cSPL with V8 was performed at $50 \mathrm{~nm} \mathrm{~s}^{-1}$, thus it appears that having 200 -fold less catalyst requires only a 20 -fold decrease in 
writing speed to perform a broadly similar end result - the creation of a micron-scale square amenable to subsequent fluorescent labelling showing high contrast against the background.

Comparisons between atomic and enzymatic catalysts on probes is further complicated by the fact that the actual site of catalysis remains unclear and desorbed metal atoms may in fact be the key catalytic species and the lithography is not be strictly confined to the probe-surface interface. Davis et al. ${ }^{33}$ proposed the PVP-PdNPs employed on their probes produced a diffuse 'cloud' of Pd atoms complexed to the PVP shell. Evidence for this leaching of metals from the probe is offered by the fact that high levels of background catalysis could be prevented with the addition of chelators to scavenge any leached metals. ${ }^{\mathbf{1 6}}$ Furthermore, similar systems employing Pd-coated $\mu \mathrm{CP}$ stamps have shown transfer of Pd to the surface. ${ }^{34}$

Pre-treatment of both the probes prior to nanolithography is also influential. For example, annealing of $\mathrm{TiO}_{2}$-coated probes ${ }^{24}$ promotes phase transition of $\mathrm{TiO}_{2}$ to the more catalytically active anatase phase. Overnight air curing of $\mathrm{Cu}$-coated probes ${ }^{19}$ yielded substantial improvements in activity, whilst Cu-coated $\mu \mathrm{CP}$ stamps were measured to be 5 times more active following similar overnight curing, ${ }^{36}$ likely due to the formation of a native oxide layer containing catalytically active $\mathrm{Cu}(\mathrm{I})$ ions. Preparation of the surfaces prior to nanolithography is also an important factor. Vacuum curing of the siloxane $\mathrm{SAMs}^{\mathbf{1 6}}$ was shown to improve patterning resolution (from $200 \mathrm{~nm}$ to $30-40$ $\mathrm{nm}$ ) through the promotion of silanol condensation, improving monolayer ordering and stability, permitting writing to occur at greater loading forces (thus a greater rate of catalysis) before displacement of the SAM occurs.

A more practical consideration is the limited lifespan of immobilised catalysts. Wherever tested, both cSPL probes ${ }^{21,28,33}$ and $\mu \mathrm{CP}$ stamps $\mathrm{s}^{\mathbf{3 4 , 3 6 , 4 5 , 4 7}}$ were found have lost most or all of their activity with repeated use (typically 10 's $\mu \mathrm{m}^{2}$ of scanned area for cSPL probes and 4-6 uses for catalytic $\mu \mathrm{CP}$ stamps). For metallic coatings, it appears that the loss of activity is not simply due to the absolute loss of catalyst due to leaching but also the loss of highly reactive catalytic sites, possibly due to Ostwald ripening (see Section 2.2 above). In the case of enzymes, it seems likely that the loss of activity is due to the unfolding (denaturation) of the enzyme under the high shear forces and pressures generated by the moving probe.

\section{Conclusions and outlook}

There have now been many reports that convincingly demonstrate the chemical scope of cSPL and the flexibility of combining chemical specificity of catalysis with the positional specificity of SPM.

However, the vast majority of the reported examples are proof-of-concept studies. In order for these methods to be more widely applied to practical applications, a number of issues remain to be addressed such as the limited probe lifespan and more convenient probe functionalisation methods in the case of molecular or enzymatic catalysts. Detailed studies of the physical conditions present at the probe tip will also be crucial to understanding the mechanisms of catalysis and experimental parameter optimisation, which in turn are necessary for the development of improved cSPL systems. For example, uncertainties regarding the temperatures generated during writing could be measured by the use of thermocouple AFM probes, which have been fabricated with both Pt and Pd as the thermocouple junctions on the probe apex. ${ }^{\mathbf{4 8 , 4 9}}$ As such they would be catalytically active across a range of surface chemistries and would allow in situ monitoring of the temperature.

Looking to the future, parallelisation through the use of arrays of multiple probes (e.g. through PPL or HSL) to enable higher lithographic throughput and large-area fabrication is one clear direction for maturation of the technology, particularly in nanoelectronics production. In a similar manner, beam pen lithography and the Snomipede offer future routes towards the multiplexing of photocatalytic reactions. ${ }^{\mathbf{5 0 , 5 1}}$

Spatial resolution may be substantially enhanced by the use of single enzymes or single NPs at the probe apex. For example, methods already exist for single NPs to be synthesised at AFM probe apices. ${ }^{52}$ In the case of enzymatic cSPL, there are now powerful directed evolution methods that enable the rapid generation of highly active enzymes. ${ }^{53}$ Such genetic recombinant methods could be utilised to generate and select enzymes specifically for cSPL applications. Biocatalytic cSPL would also benefit from harnessing improved site-specific enzyme immobilisation methods, ${ }^{54,55}$ to ensure that the enzymes attached to the probes retain maximum activity. This aspect is particularly crucial for nanoscale applications where relatively few protein molecules can be located on a single nanoscale object. A conceptually interesting but more speculative avenue would be the use of diffusion-limited enzymes ${ }^{56,57}$ (where catalysis occurs so rapidly the reaction rate is limited by the rate of diffusion of reactants) would make excellent candidates for probe mounted catalysts, as the enzyme could be presented with substrate at a rate greater than that possible by diffusion, potentially enabling extremely rapid lithography.

In summary catalytic scanning probe lithography combines the chemical specificity of catalysis with the positional specificity of SPM, and has achieved patterning precisions approaching those of many classical SPL techniques. Although there are still obstacles to be overcome, with refinements to throughput, resolution and probe lifespan, the technique potentially represents a disruptive manufacturing technology and a step towards an efficient, compact and universal "desktop fab".

\section{Acknowledgements}

The authors acknowledge financial support from the UK Engineering and Physical Sciences Research Council (EP/K011685/1, $\mathrm{EP} / \mathrm{K} 024485 / 1)$.

\section{Notes and references}

1 B. D. Gates, Q. Xu, M. Stewart, D. Ryan, C. G. Willson and G. M. Whitesides, Chem. Rev., 2005, 105, 1171-1196. 
2 H. Sugihara, A. Takahara and T. Kajiyama, J. Vac. Sci. Technol., B: Microelectron. Nanometer Struct.-Process., Meas., Phenom., 2001, 19, 593-595.

3 S. Xu and G.-y. Liu, Langmuir, 1997, 13, 127-129.

4 H. C. Day and D. R. Allee, Appl. Phys. Lett., 1993, 62, 26912693.

5 W.-K. Lee, M. Haydell, J. T. Robinson, A. R. Laracuente, E. Cimpoiasu, W. P. King and P. E. Sheehan, ACS Nano, 2013, 7, 6219-6224.

6 L. R. Giam and C. A. Mirkin, Angew. Chem., Int. Ed., 2011, 50, 7482-7485.

7 K. Salaita, Y. Wang and C. A. Mirkin, Nat. Nanotechnol., 2007, 2, 145-155.

8 L. R. Giam, A. J. Senesi, X. Liao, L. S. Wong, J. Chai, D. J. Eichelsdoerfer, W. Shim, B. Rasin, S. He and C. A. Mirkin, Proc. SPIE, 2011, 8031, 803103.

9 A. Lewis, Y. Kheifetz, E. Shambrodt, A. Radko, E. Khatchatryan and C. Sukenik, Appl. Phys. Lett., 1999, 75, 2689-2691.

10 S. Asmahan, K. Wonmo, C. David, D. Ralu, M. Nicolae and D. E. Horacio, J. Micromech. Microeng., 2013, 23, 125014.

11 J. Hyun, J. Kim, S. L. Craig and A. Chilkoti, J. Am. Chem. Soc., 2004, 126, 4770-4771.

12 B. Basnar, Y. Weizmann, Z. Cheglakov and I. Willner, Adv. Mater., 2006, 18, 713-718.

13 D. A. Long, K. Unal, R. C. Pratt, M. Malkoch and J. Frommer, Adv. Mater., 2007, 19, 4471-4473.

14 H. Li, Q. He, X. Wang, G. Lu, C. Liusman, B. Li, F. Boey, S. S. Venkatraman and H. Zhang, Small, 2011, 7, 226229.

15 W. T. Müller, D. L. Klein, T. Lee, J. Clarke, P. L. McEuen and P. G. Schultz, Science, 1995, 268, 272-273.

16 C. Blackledge, D. A. Engebretson and J. D. McDonald, Langmuir, 2000, 16, 8317-8323.

17 J. J. Davis, K. S. Coleman, K. L. Busuttil and C. B. Bagshaw, J. Am. Chem. Soc., 2005, 127, 13082-13083.

18 J. J. Davis, C. B. Bagshaw, K. L. Busuttil, Y. Hanyu and K. S. Coleman, J. Am. Chem. Soc., 2006, 128, 14135-14141.

19 W. F. Paxton, J. M. Spruell and J. F. Stoddart, J. Am. Chem. Soc., 2009, 131, 6692-6694.

20 K. Zhang, Q. Fu, P. Nan, X. Yu, J. Liu, Y. Luo, X. Wang, J. Yang and J. Hou, Nat. Commun., 2012, 3, 1194.

21 D. A. Valyaev, S. Clair, L. Patrone, M. Abel, L. Porte, O. Chuzel and J.-L. Parrain, Chem. Sci., 2013, 4, 2815-2821.

22 M. Péter, X.-M. Li, J. Huskens and D. N. Reinhoudt, J. Am. Chem. Soc., 2004, 126, 11684-11690.

23 V. Zorbas, M. Kanungo, S. A. Bains, Y. Mao, T. HemrajBenny, J. A. Misewich and S. S. Wong, Chem. Commun., 2005, 4598-4600.

24 E. Ul-Haq, S. Patole, M. Moxey, E. Amstad, C. Vasilev, C. N. Hunter, G. J. Leggett, N. D. Spencer and N. H. Williams, ACS Nano, 2013, 7, 7610-7618.

25 S. Takeda, C. Nakamura, C. Miyamoto, N. Nakamura, M. Kageshima, H. Tokumoto and J. Miyake, Nano Lett., 2003, 3, 1471-1474.

26 C. Nakamura, C. Miyamoto, I. Obataya, N. Nakamura and J. Miyake, Proc. SPIE, 2004, 5593, 277-283.
27 L. Riemenschneider, S. Blank and M. Radmacher, Nano Lett., 2005, 5, 1643-1646.

28 C. Nakamura, C. Miyamoto, I. Obataya, S. Takeda, M. Yabuta and J. Miyake, Biosens. Bioelectron., 2007, 22, 2308-2314.

29 X. Luo, V. A. Pedrosa and J. Wang, Chem.-Eur. J., 2009, 15, 5191-5194.

30 R. A. W. Johnstone, A. H. Wilby and I. D. Entwistle, Chem. Rev., 1985, 85, 129-170.

31 Z. Wei, D. Wang, S. Kim, S.-Y. Kim, Y. Hu, M. K. Yakes, A. R. Laracuente, Z. Dai, S. R. Marder, C. Berger, W. P. King, W. A. de Heer, P. E. Sheehan and E. Riedo, Science, 2010, 328, 1373-1376.

32 D. Yang, A. Velamakanni, G. Bozoklu, S. Park, M. Stoller, R. D. Piner, S. Stankovich, I. Jung, D. A. Field, C. A. Ventrice, Jr and R. S. Ruoff, Carbon, 2009, 47, 145-152. 33 J. J. Davis and Y. Hanyu, Nanotechnology, 2010, 21, 265302.

$34 \mathrm{H}$. Mizuno and J. M. Buriak, ACS Appl. Mater. Interfaces, 2010, 2, 2301-2307.

35 H. Mizuno and J. M. Buriak, J. Am. Chem. Soc., 2008, 130, 17656-17657.

36 J. M. Spruell, B. A. Sheriff, D. I. Rozkiewicz, W. R. Dichtel, R. D. Rohde, D. N. Reinhoudt, J. F. Stoddart and J. R. Heath, Angew. Chem., Int. Ed., 2008, 47, 9927-9932.

37 H.-Y. Chen, M. Hirtz, X. Deng, T. Laue, H. Fuchs and J. Lahann, J. Am. Chem. Soc., 2010, 132, 18023-18025.

38 S. Oberhansl, M. Hirtz, A. Lagunas, R. Eritja, E. Martinez, H. Fuchs and J. Samitier, Small, 2012, 8, 541-545.

39 S. Bian, J. He, K. B. Schesing and A. B. Braunschweig, Small, 2012, 8, 2000-2005.

40 A. A. Shestopalov, R. L. Clark and E. J. Toone, Nano Lett., 2009, 10, 43-46.

41 A. A. Shestopalov, R. L. Clark and E. J. Toone, Langmuir, 2009, 26, 1449-1451.

42 M. Ganesh, J. Nachman, Z. Mao, A. Lyons, M. Rafailovich and R. Gross, Biomacromolecules, 2013, 14, 2470-2476.

43 R. E. Ionescu, R. S. Marks and L. A. Gheber, Nano Lett., 2003, 3, 1639-1642.

44 R. E. Ionescu, R. S. Marks and L. A. Gheber, Nano Lett., 2005, 5, 821-827.

45 A. 1. Guyomard-Lack, N. Delorme, C. 1. Moreau, J.-F. o. Bardeau and B. Cathala, Langmuir, 2011, 27, 76297634.

46 P. W. Snyder, M. S. Johannes, B. N. Vogen, R. L. Clark and E. J. Toone, J. Org. Chem., 2007, 72, 7459-7461.

47 X.-M. Li, M. Péter, J. Huskens and D. N. Reinhoudt, Nano Lett., 2003, 3, 1449-1453.

48 K. Luo, Z. Shi, J. Lai and A. Majumdar, Appl. Phys. Lett., 1996, 68, 325-327.

49 K. Luo, Z. Shi, J. Varesi and A. Majumdar, J. Vac. Sci. Technol., B: Microelectron. Nanometer Struct.-Process., Meas., Phenom., 1997, 15, 349-360.

50 F. Huo, G. Zheng, X. Liao, L. R. Giam, J. Chai, X. Chen, W. Shim and C. A. Mirkin, Nat. Nanotechnol., 2010, 5, 637640.

51 E. ul-Haq, Z. Liu, Y. Zhang, S. A. Alang Ahmad, L. S. Wong, J. K. Hobbs, G. J. Leggett, J. Micklefield, C. J. Roberts and J. M. R. Weaver, J. Mater. Res., 2011, 26, 2997-3008. 
52 H. Wang, T. Tian, Y. Zhang, Z. Pan, Y. Wang and Z. Xiao, Langmuir, 2008, 24, 8918-8922.

53 M. T. Reetz, J. Biomed. Sci. Eng., 2011, 50, 138-174.

54 L. S. Wong, F. Khan and J. Micklefield, Chem. Rev., 2009, 109, 4025-4053.
55 L. S. Wong, C. V. Karthikeyan, D. J. Eichelsdoerfer, J. Micklefield and C. A. Mirkin, Nanoscale, 2012, 4, 659-666. 56 S.-X. Lin and J. Lapointe, J. Biomed. Sci. Eng., 2013, 6, 435-442. 57 G. Zhou, M.-T. Wong and G.-Q. Zhou, Biophys. Chem., 1983, 18, 125-132. 